\title{
SINGULAR COTANGENT MODEL
}

\author{
CARLOS CURRÁS-BOSCH \\ Departament d'Àlgebra i Geometria, Facultat de Matemàtiques, \\ Universitat de Barcelona, Gran Via 585, \\ 08007 Barcelona, Spain \\ e-mail: carloscurrasbosch@ub.edu
}

(Received 11 June 2013; accepted 14 January 2014; first published online 18 December 2014)

\begin{abstract}
Any singular level of a completely integrable system (c.i.s.) with nondegenerate singularities has a singular affine structure. We shall show how to construct a simple c.i.s. around the level, having the above affine structure. The cotangent bundle of the desingularized level is used to perform the construction, and the c.i.s. obtained looks like the simplest one associated to the affine structure. This method of construction is used to provide several examples of c.i.s. with different kinds of non-degenerate singularities.
\end{abstract}

Mathematics Subject Classification. 53D05, 37J35, 37G05

1. Introduction. Let $\left(M^{2 n}, \omega, F\right)$ be a non-degenerate integrable system. This means that $\left(M^{2 n}, \omega\right)$ is a symplectic manifold and $F=\left(f_{1}, \ldots, f_{n}\right)$ is a proper moment map, which is non-singular almost everywhere, and its singularities are of Morse-Bott type.

The $\mathbb{R}^{n}$-action generated by the Hamiltonian vector fields $H_{f_{1}}, \ldots, H_{f_{n}}$ gives a singular Lagrangian foliation on $\left(M^{2 n}, \omega\right)$. Any leaf is an orbit of this actions. At the same time, any connected component of $F^{-1}(c)$ is a level. We know that the regular levels are $n$-dimensional tori and the singular levels are finite union of several leaves.

A semilocal classification of such integrable systems is still open. It consists in finding a complete system of invariants describing symplectically a neighborhood of a level. Some approaches to solve this question has been currently made, see $[4,13$, 15-17]. As the local description of non-degenerate singularities is given in terms of products of elliptic, hyperbolic and focus-focus components, the number of elliptic, hyperbolic and focus-focus components at each point of the level will play an essential role in the classification problem.

As in the regular case, the Hamiltonian vector fields $H_{f_{1}}, \ldots, H_{f_{n}}$ endow any leaf and any level with an affine structure with singularities. In studying the semi-local classification, we have seen that this affine structure gives strong conditions on the set of invariants that we have found. Our proposal in this paper is to prove that from this affine structure we can construct a completely integrable system around a given level $L_{0}$ of $\left(M^{2 n}, \omega, F\right)$, such that the induced affine structure on $L_{0}$ is the given one. This construction looks like the simplest one with the given affine structure on $L_{0}$.

As the ' 1 -jet' of the former completely integrable system (c.i.s. from now on) and the one constructed in this way coincide, it makes sense to denote this c.i.s. as the linearized c.i.s. of the initial one. 
This process of construction of the linearized c.i.s. indicates us a way to construct c.i.s. with prescribed non-degenerate singularities along a given singular level. Some examples of construction are given.

I would like to express my gratitude to Pierre Molino for interesting and fruitful conversations on this subject.

\section{Definition and basic properties.}

2.1. Local expressions. Let $\left(M^{2 n}, \omega, f_{1}, \ldots, f_{n}\right)$ be an integrable system, i.e. $\left(M^{2 n}, \omega\right)$ is a symplectic manifold. The functions $f_{1}, \ldots, f_{n}$ are Poisson commuting (first integrals of a given Hamiltonian system), such that $d f_{1} \wedge \cdots \wedge d f_{n} \neq 0$ on a dense subset of $M^{2 n}$, and the moment map $F: M^{2 n} \longrightarrow \mathbb{R}^{n}, F=\left(f_{1}, \ldots, f_{n}\right)$, is proper.

Such an integrable system is said to be non-degenerate if, in a neighborhood of each point $p_{0} \in M^{2 n}$, there exist canonical coordinates $\left(x_{1}, y_{1}, \ldots, x_{n}, y_{n}\right)$, and $n$ local functions $h_{1}, \ldots, h_{n}$, which have one of the following expressions:

$$
\begin{aligned}
h_{i}= & y_{i} \quad(\text { regular terms }), \\
h_{i}= & \left(x_{i}\right)^{2}+\left(y_{i}\right)^{2} \quad \text { (elliptic terms) } \\
h_{i}= & x_{i} y_{i} \quad(\text { hyperbolic terms), } \\
& \left\{\begin{array}{l}
h_{i}=x_{i} y_{i}+x_{i+1} y_{i+1} \\
h_{i+1}=x_{i} y_{i+1}-y_{i} x_{i+1}
\end{array} \quad\right. \text { (focus-focus terms), }
\end{aligned}
$$

such that:

(1) $f_{1}, \ldots, f_{n}$ Poisson commute with $h_{1}, \ldots, h_{n}$.

(2) $\left\{j_{p_{0}}^{2} f_{1}, \ldots, j_{p_{0}}^{2} f_{n}\right\}$ and $\left\{j_{p_{0}}^{2} h_{1}, \ldots, j_{p_{0}}^{2} h_{n}\right\}$ generate the same space of 2 -jets at $p_{0}$.

This notion of non-degeneracy implies obvious conditions on the space of 2jets generated at each point by $f_{1}, \ldots, f_{n}$. Conversely, assuming these infinitesimal conditions, the existence of such adapted coordinates is an important result, due to H. Eliasson [9]. The demonstration has been completed by E. Miranda [13] and E. Miranda \& V. N. San [14]. Adapted coordinates will be referred to as Eliasson coordinates, or simply E-coordinates.

We denote as $\mathcal{L}$ the Lagrangian singular foliation associated to this c.i.s., i.e. the leaves of $\mathcal{L}$ are the orbits of the $\mathbb{R}^{n}$-action generated by $H_{f_{1}}, \ldots, H_{f_{n}}$.

From a practical point of view, one can locally look at $\left(M^{2 n}, \omega, \mathcal{L}\right)$ as $\left(\mathbb{R}^{2 n}, \omega_{0}, \mathcal{L}_{0}\right)$, where $\omega_{0}$ is the standard symplectic two-form on $\mathbb{R}^{2 n}$ and $\mathcal{L}_{0}$ is given by $d h_{i}=0$, $i=1, \ldots, n$.

Let $L_{0}$ be a singular level, and $p_{0}$ a point of $L_{0}$. By taking E-coordinates around $p_{0}$ we have the following characteristic numbers of the point $p_{0}$ : the numbers $k_{e}, k_{h}$ and $k_{f}$ correspond to the number of elliptic, hyperbolic and focus-focus terms of the set $\left(h_{1}, \ldots, h_{n}\right)$. The leaf through $p_{0}$ is $\mathbb{T}^{c} \times \mathbb{R}^{o}$, and the numbers $c$ and $o$ are called the degrees of closedness and openness of the leaf, respectively.

Following Zung (see [20]), the 5-tuple $\left(k_{e}, k_{h}, k_{f}, c, o\right)$ is called the leaf-type and $\left(k_{e}, k_{h}, k_{f}\right)$ the Williamson type of $p_{0}$. In general one has $k_{e}+k_{h}+2 k_{f}+c+o=n$. In [20] it is proved that the three numbers $k_{e}, k_{f}+c, k_{h}+k_{k}+o$, are invariants of each level. These numbers are known as the degrees of ellipticity, closedness and openness of the level. 
Summarizing, one can say that a singular level $L_{0}$ is a compact $\left(n-k_{e}\right)$-manifold, with self-intersections provided with a manifold structure of dimension less than $(n-$ $k_{e}$ ). This level is endowed with a non-degenerate $\mathbb{R}^{n-k_{e}}$-action. Non-degenerate action means that the isotropy of this action at each point is linearizable.

Let $L_{0}$ be a singular level of a c.i.s. $\left(M^{2 n}, \omega, F\right)$; it is known that the degree of ellipticity $k_{e}$ is the same at all the points of $L_{0}$. Associated to this degree of ellipticity, we have a Hamiltonian $\mathbb{T}^{k_{e}}$-action in a neighborhood of $L_{0}$, such that the isotropy of this action contains $L_{0}$. Due to this, one can see, using for instance coordinates adapted to this $\mathbb{T}^{k_{e}}$-action, that the isotropy subset of this action is a symplectic submanifold $M^{2\left(n-k_{e}\right)} \subset M^{2 n}$. This symplectic submanifold is obviously a neighborhood of $L_{0}$ and one has an induced c.i.s. on $M^{2\left(n-k_{e}\right)}$ such that $L_{0}$ is a singular level.

From this point, we can regard $L_{0}$ as a singular level in the c.i.s. defined on $M^{2\left(n-k_{e}\right)}$, The degree of ellipticty in $L_{0}$ is obviously zero. So, from now on we shall restrict our attention to the case of singular levels with $k_{e}=0$. Once obtained the linearized model in this case, a simple product with $k_{e}$ copies of the standard model in the unit disk $E_{2}$ will produce, in general, the model that we are searching for.

2.2. Desingularized level. We assume that on the singular level $L_{0}$, with singular affine structure $\nabla_{0}$, the degree of ellipticity vanishes. In order to give a construction of a 'standard' c.i.s., such that $L_{0}$ is a singular level, and the induced singular affine structure on it coincides with $\nabla_{0}$, we start by giving the construction of the so called desingularized level.

As $k_{e}=0$ on $L_{0}$, dim. $L_{0}=n$. Let $\psi$ be a differentiable embedding of $M^{2 n}$ in an euclidean space $\mathbb{R}^{l}$. Let $L_{0}^{r} \subset L_{0}$ be the subset of regular points of $L_{0}$, i.e., a point $p \in L_{0}$ lies in $L_{0}^{r}$ if and only if the rank of $d F$ is equal to $n$ at $p$. Note that $L_{0}^{r}$ is not, in general, a connected submanifold.

Let $G_{n}\left(\mathbb{R}^{l}\right)$ be the $n$-dimensional Grassmann manifold of $\mathbb{R}^{l}$. For any $n$-dimensional vector subspace $V$ of $\mathbb{R}^{l}$, let $[V]$ be corresponding class in $G_{n}\left(\mathbb{R}^{l}\right)$.

We consider the following embedding of $L_{0}^{r}$ in $M^{2 n} \times G_{n}\left(\mathbb{R}^{l}\right)$ given by:

$$
\varphi: L_{0}^{r} \hookrightarrow M^{2 n} \times G_{n}\left(\mathbb{R}^{l}\right), \quad p \mapsto\left(p,\left[T_{\psi(p)}\left(\psi\left(L_{0}\right)\right)\right]\right)
$$

We define the desingularized level, $\hat{L}_{0}$ as the closure of $\varphi\left(L_{0}^{r}\right)$ in $M^{2 n} \times G_{n}\left(\mathbb{R}^{l}\right)$.

Claim 2.1. $\hat{L}_{0}$ is a $n$-dimensional submanifold of $M^{2 n} \times G_{n}\left(\mathbb{R}^{l}\right)$

Proof. Let $q$ be a point of $\varphi\left(L_{0}^{r}\right)$. As $\varphi$ is an embedding, we have a $n$-dimensional natural chart defined around $q$.

Let $q=(p,[V])$ be a point in $\mathrm{Cl}\left(\varphi\left(L_{0}^{r}\right)\right) \backslash \varphi\left(L_{0}^{r}\right)$. Obviously $p \in L_{0} \backslash L_{0}^{r}$. We have to provide a system of coordinates in a neighborhood of $(p,[V])$. Let $\left(k_{h}, k_{f}\right)$ be the Williamsom type of the point $p$. We know that there is a chart $U$ in $M^{2 n}$, with coordinates $\left(x_{1}, y_{1}, \ldots, x_{n}, y_{n}\right)$, centered at $p$, and $L_{0} \cap U$ is given by $h_{i}=0$, $i=1, \ldots, n$, where the functions $h_{i}$ are in the form of Section 2.1. Let us have a look at the tangent space to $L_{0}$ at a point near to $p$ : from the expressions of the functions $h_{i}$, we see

$$
L_{0} \cap U=\prod_{j=1}^{s}\left(L_{0}^{j} \cap U^{j}\right),
$$


where each $L_{0}^{j} \cap U^{j}$ is of the form

(a) $U^{j}=D_{2}$, with coordinates $\left(x_{j}, y_{j}\right), h_{j}=y_{j}$, and $L_{0}^{j} \cap U^{j}$ is given by $h_{j}=0$. These are the regular terms, and there are $n-k_{h}-2 k_{f}$ regular terms.

(b) $U^{j}=D_{2}$, with coordinates $\left(x_{j}, y_{j}\right), h_{j}=x_{j} y_{j}$, and $L_{0}^{j} \cap U^{j}$ is given by $h_{j}=0$ (hyperbolic term).

(c) $U_{j}=D_{2} \times D_{2}$, with coordinates $\left(x_{j}, y_{j}, x_{j+1}, y_{j+1}\right), h_{j}=x_{j} y_{j}+x_{j+1} y_{j+1}, h_{j+1}=$ $x_{j} y_{j+1}-x_{j+1} y_{j}$, and $L_{0}^{j} \cap U^{j}$ is given by $h_{j}=h_{j+1}=0$ (focus-focus term).

According to this, we give a system of coordinates around $(p,[V])$, by considering the above three possibilities:

In the case (a)

$$
T_{\left(x_{j}, 0\right)}\left(L_{0}^{j} \cap U^{j}\right)=\left\langle\frac{\partial}{\partial x_{j}}\right\rangle,
$$

where $\left\langle\frac{\partial}{\partial x_{j}}\right\rangle$ means the vector subspace generated by $\frac{\partial}{\partial x_{j}}$.

In this case $L_{0}^{j} \cap U^{j}$ is a regular submanifold,

$$
T_{(0,0)}\left(L_{0}^{j} \cap U^{j}\right)=\left\langle\frac{\partial}{\partial x_{j}}\right\rangle
$$

so, $\left\langle\frac{\partial}{\partial x_{j}}\right\rangle \subset V$ and we take the function $x_{j}$ as the coordinate, coming from the regular term, in a neighborhood of $(p,[V])$.

In the case (b)

$$
T_{\left(0, y_{j}\right)}\left(L_{0}^{j} \cap U^{j}\right)=\left\langle\frac{\partial}{\partial y_{j}}\right\rangle
$$

or

$$
T_{\left(x_{j}, 0\right)}\left(L_{0}^{j} \cap U^{j}\right)=\left\langle\frac{\partial}{\partial x_{j}}\right\rangle .
$$

At the point $(0,0) \in\left(L_{0}^{j} \cap U^{j}\right)$ we have $\left\langle\frac{\partial}{\partial y_{j}}\right\rangle \subset V$ or $\left\langle\frac{\partial}{\partial x_{j}}\right\rangle \subset V$, so $y_{j}$ or $x_{j}$ will be used as the coordinate, coming from the hyperbolic term, in a neighborhood of $(p,[V])$. In the case (c)

$$
\begin{gathered}
T_{\left(0, y_{j}, 0, y_{j+1}\right)}\left(L_{0}^{j} \cap U^{j}\right)=\left\langle\frac{\partial}{\partial y_{j}}, \frac{\partial}{\partial y_{j+1}}\right\rangle, \\
\text { or } \quad T_{\left(x_{j}, 0, x_{j+1}, 0\right)}\left(L_{0}^{j} \cap U^{j}\right)=\left\langle\frac{\partial}{\partial x_{j}}, \frac{\partial}{\partial x_{j+1}}\right\rangle,
\end{gathered}
$$

at the point $(0,0,0,0) \in\left(L_{0}^{j} \cap U^{j}\right)$ we have $\left\langle\frac{\partial}{\partial y_{j}}, \frac{\partial}{\partial y_{j+1}}\right\rangle \subset V$ or $\left\langle\frac{\partial}{\partial x_{j}}, \frac{\partial}{\partial x_{j+1}}\right\rangle \subset V$, so $\left(y_{j}, y_{j+1}\right)$ or $\left(x_{j}, x_{j+1}\right)$ will be used as the coordinates, coming from the focus-focus term, in a neighborhood of $(p,[V])$.

The system of coordinates around $(p,[V])$ is the result of summing up the above coordinates, and it is quite obvious the compatibility of the charts defined in this form. 
The above considerations show us the $C^{\infty}$-structure of $\hat{L}_{0}$, and provide us good systems of coordinates to use in the sequel.

The map $j: \hat{L}_{0} \longrightarrow M^{2 n}$ given by $j(p,[V])=p$ is differentiable, and we see that $j\left(\hat{L}_{0}\right)=L_{0}$. The preimage of any singular point on $L_{0}$ consists of $2^{k_{h}+k_{f}}$ points on $\hat{L}_{0}$. This map is a Lagrangian immersion in $\left(M^{2 n}, \omega\right)$

\section{The singular cotangent model.}

3.1. Affine structure on the desingularized level. The Poisson action of the local $F$ basic functions defines a singular affine structure $\nabla_{0}$ on $L_{0}$. On each leaf of the level, the affine structure is defined by considering the infinitesimal generators of the $\mathbb{R}^{n}$-action as parallel vector fields. This $\mathbb{R}^{n}$-action can be lifted in a natural way to $\hat{L}_{0}$. Note that this lift is possible because the map $j: \hat{L}_{0} \longrightarrow M^{2 n}$ is an immersion. As the affine structure on $L_{0}$ is provided by the Hamiltonian vector fields $\left.H_{f_{1}}\right|_{L_{0}}=X_{1}, \ldots,\left.H_{f_{n}}\right|_{L_{0}}=X_{n}$ it seems natural to write as $\hat{X}_{i}, i=1, \ldots, n$, the vector fields on $\hat{L}_{0}$ induced through the immersion $j$, and $\hat{\nabla}_{0}$ this affine structure.

By using the affine structure on $\hat{L}_{0}$ we are going to construct a completely integrable system on $\left(T^{*} \hat{L}_{0}, \hat{\omega}_{0}\right)$, where $\hat{\omega}_{0}$ is the standard symplectic two-form on $T^{*} \hat{L}_{0}$, and such that the affine structure on $\hat{L}_{0}$, induced by this c.i.s. is $\hat{\nabla}_{0}$.

In an ultimate step, by a process of gluing, using natural local identifications, we will obtain a c.i.s., such that $L_{0}$ is one level, and the affine structure on $L_{0}$ will be $\nabla_{0}$.

3.2. A completely integrable system on $T^{*} \hat{L}_{0}$. We define $n$ differentiable functions $g_{1}, \ldots, g_{n}$ on $T^{*} \hat{L}_{0}$ by

$$
g_{i}((p,[V]), w):=\left\langle\hat{X}_{i}(p,[V]), w\right\rangle
$$

and we will prove that $\left(T^{*} \hat{L}_{0}, \hat{\omega}_{0},\left(g_{1}, \ldots, g_{n}\right)\right)$ is completely integrable. To do it, we need only prove that $\left.\left\{g_{i}, g_{j}\right\}\right|_{0}=0$, where $\left.\{.,\}\right|_{0$.$} means the standard Poisson bracket$ in $T^{*} \hat{L}_{0}$. By a continuity argument, it will be sufficient to prove it at the points $((p,[V]),-) \in T^{*} \hat{L}_{0}$, where $p$ is a regular point. We can take Eliasson coordinates $\left(x_{1}, y_{1}, \ldots, x_{n}, y_{n}\right)$ around the point $p$, and as any basic function only depends on $\left(y_{1}, \ldots, y_{n}\right)$ in a neighborhood of $p$, the expression of $H_{f_{i}}$ in this neighborhood will be of the form $\sum_{j=1}^{n} \frac{\partial f_{i}}{\partial y_{j}}(0, \ldots, 0) \frac{\partial}{\partial x_{j}}$, i.e. is a vector fields with constant coefficients (which is obvious because the vector field is affine parallel).

Let $\alpha$ be the Liouville form on $T^{*} \hat{L}_{0}$. In this neighborhood, $\alpha=\sum_{i=1}^{n} x_{i}^{*} d x_{i}$, $g_{i}=\sum_{k=1}^{n} x_{k}^{*} \frac{\partial f_{i}}{\partial y_{k}}(0, \ldots, 0)$, so $d g_{i}=\sum_{k=1}^{n} \frac{\partial f_{i}}{\partial y_{k}}(0, \ldots, 0) d x_{k}^{*}$, and obviously

$$
\Lambda^{0}\left(d g_{i}, d g_{j}\right)=0
$$

Once we know that $\left(T^{*} \hat{L}_{0}, \hat{\omega}_{0},\left(g_{1}, \ldots, g_{n}\right)\right)$ is completely integrable, we remark that, in general, is not proper: let us assume, for instance that $\operatorname{dim} . L_{0}=1$, and let $p$ be a hyperbolic point, we can write $\omega_{0}=d x \wedge d y$, and $h=x y$, then $\{x=0\}$ is a leaf.

For further considerations it seems suitable to look at the local expressions of the above functions $g_{i}(i=1, \ldots, n)$ : let $(p,[V])$ be a point in $\hat{L}_{0}$, where $p$ is a point of $L_{0}$ of Williamson type $\left(k_{h}, k_{f}\right)$, and let $r$ be the number of regular terms at $p$. This means 
that in a system of E-coordinates $\left(x_{1}, \ldots, y_{n}\right)$ around $p$, we have

$$
h_{i}=y_{i}, \quad i=1, \ldots, r \quad(\text { regular terms })
$$

$$
h_{i}=x_{i} y_{i}, \quad i=r+1, . ., r+k_{h} \quad \text { (hyperbolic terms), }
$$

$$
\left\{\begin{array}{l}
h_{i}=x_{i} y_{i}+x_{i+1} y_{i+1} \\
h_{i+1}=x_{i} y_{i+1}-x_{i+1} y_{i}
\end{array} \quad i=r+k_{h}+1, \ldots, r+k_{h}+2 k_{f}-1 \quad\right. \text { (focus-focus terms). }
$$

According to the proof of Claim 2.1, we can assume, without loss of generality, that

$$
V=\bigoplus_{i=1}^{r}\left\langle\frac{\partial}{\partial x_{i}}\right\rangle \bigoplus_{i=r+1}^{r+k_{h}}\left\langle\frac{\partial}{\partial x_{i}}\right\rangle_{i=r+k_{h}+1}^{r+k_{h}+2 k_{f}}\left\langle\frac{\partial}{\partial x_{i}}, \frac{\partial}{\partial x_{i+1}}\right\rangle
$$

So, the system of coordinates that we consider around $(p,[V])$ is

$$
\left(x_{1}, \ldots, x_{r} ; x_{r+1}, \ldots, x_{r+k_{h}} ; x_{r+k_{h}+1}, \ldots, x_{r+k_{h}+2 k_{f}}\right) \text {. }
$$

In the above neighborhood of $p \in M$, any function $f_{i}$ can be expressed as

$$
f_{i}=f_{i}\left(h_{1}, \ldots, h_{n}\right)
$$

so the expression of $d f_{i}$ is

$$
d f_{i}=\sum_{j=1}^{n} D_{j} f_{i} \cdot d h_{j}
$$

and as the coefficients of this last expression are constant on each level, we have that in $L_{0}$

$$
\left.H_{f_{i}}\right|_{L_{0}}=\left.\sum_{j=1}^{n} a_{j}^{(i)} \cdot H_{h_{j}}\right|_{L_{0}}, \quad a_{j}^{(i)} \in \mathbb{R}
$$

The determinant of the matrix $\left(a_{j}^{(i)}\right)$ being non zero.

Finally, in the cotangent space of the above neighborhood of $(p,[V])$, endowed with canonical coordinates

$$
\left(x_{1}, x_{1}^{*}, \ldots, x_{r}, x_{r}^{*} ; x_{r+1}, x_{r+1}^{*}, \ldots ; x_{r+k_{h}+1}, x_{r+k_{h}+1}^{*}, x_{r+k_{h}+2}, x_{r+k_{h}+2}^{*}, \ldots\right)
$$

the expression of $g_{i}$ is

$$
\begin{aligned}
g_{i}= & a_{1}^{(i)} x_{1}^{*}+\cdots+a_{r+1}^{(i)} x_{r+1} x_{r+1}^{*}+\cdots+a_{r+k_{h}+1}^{(i)}\left(x_{r+k_{h}+1} x_{r+k_{h}+1}^{*}+x_{r+k_{h}+2} x_{r+k_{h}+2}^{*}\right) \\
& +a_{r+k_{h}+2}^{(i)}\left(x_{r+k_{h}+1} x_{r+k_{h}+2}^{*}-x_{r+k_{h}+2} x_{r+k_{h}+1}^{*}\right) .
\end{aligned}
$$


3.3. The singular cotangent model. In order to have a proper completely integrable system around the level $L_{0}$ we have to define appropriate identifications between the germs of $T^{*} \hat{L}_{0}$ at the different preimages $j^{-1}(p)$ for any singular point $p \in L_{0}$, and we will obtain a germ of c.i.s. around $L_{0}$. To do it, we start by describing a neighborhood in $L_{0}$ of any singular point. We shall see that such a neighborhood admits a canonical decomposition as product of three terms, one corresponding to the regular part, one corresponding to the hyperbolic terms and the third one corresponding to the focus-focus terms.

Let $p$ be a singular point of rank $r=n-\left(k_{h}+2 k_{f}\right)$. We know that there is a system of E-coordinates around $p$, and centered at $p$,

$$
\left(x_{1}, \ldots, x_{r}, y_{1}, \ldots, y_{r} ; x_{r+1}, y_{r+1}, \ldots ; x_{r+k_{h}+1}, x_{r+k_{h}+2}, y_{r+k_{h}+1}, y_{r+k_{h}+2}, \ldots\right)
$$

such that $\left(x_{1}, \ldots, x_{r}, y_{1}, \ldots, y_{r}\right)$ corresponds to the regular part, $\left(x_{r+1}, y_{r+1}\right)$, $\ldots,\left(x_{r+k_{h}}, y_{r+k_{h}}\right)$ are associated to the hyperbolic branches, and $\left(x_{r+k_{h}+1}, x_{r+k_{h}+2}, y_{r+k_{h}+1}, y_{r+k_{h}+2}\right), \ldots,\left(x_{r+k_{h}+2 k_{f}-1}, x_{r+k_{h}+2 k_{f}}, y_{r+k_{h}+2 k_{f}-1}, y_{r+k_{h}+2 k_{f}}\right)$ correspond to the focus-focus branches.

We point out the following important remark: for any two systems of E-coordinates around the same point $p$, one can see that the axes through $p$, given by the following expressions, do not depend on the chosen system of $\mathbf{E}$-coordinates around $p$. These axes are given by

$$
\begin{array}{ccc}
\left\{\left(0, \ldots, 0, x_{r+1}, 0, \ldots, 0\right)\right\} & \cup & \left.\cup\left(0, \ldots, 0,0, y_{r+1}, 0, \ldots, 0\right)\right\} \\
& \ldots \ldots \\
\left\{\left(0, \ldots, 0, x_{r+k_{h}}, 0, \ldots, 0\right)\right\} & \cup \quad\left\{\left(0, \ldots, 0, y_{r+k_{h}}, 0, \ldots, 0\right)\right\}
\end{array}
$$

these pairs of axes correspond to the hyperbolic components.

To see it one can observe that if $\left\{x_{1}^{\prime}, y_{1}^{\prime}, \ldots\right\}$ is another system of E-coordinates around $p$. We can assume that a local affine parallel vector field like

$$
X^{\prime}=x_{r+1}^{\prime} \frac{\partial}{\partial x_{r+1}^{\prime}}-y_{r+1}^{\prime} \frac{\partial}{\partial y_{r+1}^{\prime}}
$$

restricted to the level through $p$ is expressed by a linear combination with more than one hyperbolic or focus-focus affine parallel vector field, let us assume, for instance, that its expression on the level is

$$
X^{\prime}=a\left(x_{i} \frac{\partial}{\partial x_{i}}-y_{i} \frac{\partial}{\partial y_{i}}\right)+b\left(x_{j} \frac{\partial}{\partial x_{j}}-y_{j} \frac{\partial}{\partial y_{j}}\right), \quad i, j=r+1, \ldots, r+k_{h}
$$

for some non-zero constants $a$ and $b$. By considering the locus of points where the vector field vanishes we see that necessarily $a$ or $b$ must be zero. A similar argument will exclude expressions of the type focus-focus in a decomposition like the above one. So the axes above described, and characterized by the fact that the point $p$ is in the closure of the orbits of such vector field, so do not depend on the chosen E-coordinates.

We have just seen that any local affine vector field of the form

$$
X^{\prime}=x_{j}^{\prime} \frac{\partial}{\partial x_{j}^{\prime}}-y_{j}^{\prime} \frac{\partial}{\partial y_{j}^{\prime}}, \quad j=r+1, \ldots, r+k_{h}
$$


restricted to $L_{0}$ verifies

$$
X^{\prime}=a_{l}\left(x_{l} \frac{\partial}{\partial x_{l}}-y_{l} \frac{\partial}{\partial y_{l}}\right) \quad\left(\text { for a constant } a_{l}\right), \quad l=r+1, \ldots k_{h} .
$$

Summarizing this information for all the hyperbolic components, we have seen that on the level $L_{0}$, and after a reordering of the hyperbolic components, the relation between the corresponding hyperbolic E-coordinates is

$$
x_{i}^{\prime}=b_{i} x_{i}^{\frac{1}{a_{i}}}, y_{i}^{\prime}=c_{i} y_{i}^{\frac{1}{a_{i}}}, \quad i=r+1, \ldots, r+k_{h},
$$

where $a_{i}, b_{i}, c_{i}$ are constants. Looking at the expression of $d x_{i} \wedge d y_{i}$ which must coincide with $d x_{i}^{\prime} \wedge d y_{i}^{\prime}$ when $x_{i}=y_{i}=x_{i}^{\prime}=y_{i}^{\prime}=0$, we see that $a_{i}=1$ and $b_{i}=\frac{1}{c_{i}}$.

In order to attain the main result, we will use that on $L_{0}$ the coordinates $x_{i}^{\prime}, y_{i}^{\prime}$ only depend on $x_{i}, y_{i},\left(i=r+1, \ldots, r+k_{h}\right)$, respectively.

Now we focus our study on the focus-focus part, and we shall give a similar result. We shall prove that the pairs of planes

$$
\begin{aligned}
\left\{\left(0, \ldots, 0, x_{r+k_{h}+1}, x_{r+k_{h}+2}, 0, \ldots, 0\right)\right\} & \cup\left\{\left(0, \ldots, 0, y_{r+k_{h}+1}, y_{r+k_{h}+2}, 0 \ldots, 0\right)\right\} \\
& \ldots \\
\left.\left\{0, \ldots, 0, x_{r+k_{h}+2 k_{f}-1}, 0, x_{r+k_{h}+2 k_{f}}, 0\right)\right\} & \cup\left\{\left(0, \ldots, 0, y_{r+k_{h}+2 k_{f}-1}, 0, y_{r+k_{h}+2 k_{f}}\right)\right\}
\end{aligned}
$$

do not depend on the chosen E-coordinates. The proof is quite similar to the above one. We just point out the main steps of the proof: recall that for each component of the focus-focus part given by

$$
\begin{aligned}
& \left\{\left(0, \ldots, 0, x_{l}, x_{l+1}, 0, \ldots, 0\right)\right\} \cup\left\{\left(0, \ldots, 0, y_{l}, y_{l+1}, 0 \ldots, 0\right)\right\}, \\
& l=r+k_{h}+1, \ldots, r+k_{h}+2 k_{f}-1
\end{aligned}
$$

the corresponding local basic functions are

$$
h_{l}=x_{l} y_{l}+x_{l+1} y_{l+1}, \quad h_{l+1}=x_{l} y_{l+1}-y_{l} x_{l+1} .
$$

By using the complex coordinates $\left(z_{l}, z_{l+1}\right)$, with $z_{l}:=x_{l}+i x_{l+1}, z_{l+1}:=y_{l}+i y_{l+1}$. We have

$$
h_{l}+i h_{l+1}=\bar{z}_{l} \cdot z_{l+1}
$$

The term of the canonical symplectic two-form is written as

$$
d x_{l} \wedge d y_{l}+d x_{l+1} \wedge d y_{l+1}=\operatorname{Re} \quad\left(d \bar{z}_{l} \wedge d z_{l+1}\right) .
$$

The Lagrangian foliation is obtained by considering the level surfaces of the function $\bar{z}_{l} \cdot z_{l+1}$.

Using this complex coordinates $\left(z_{l}, z_{l+1}\right)$, the level through the point $(0,0)$ is the union of the planes $\left\{z_{l}=0\right\}$ and $\left\{z_{l+1}=0\right\}$.

The Hamiltonian vector field $H_{h_{l}}$ is

$$
H_{h_{l}}=z_{l} \frac{\partial}{\partial z_{l}}+\bar{z}_{l} \frac{\partial}{\partial \bar{z}_{l}}-z_{l+1} \frac{\partial}{\partial z_{l+1}}-\bar{z}_{l+1} \frac{\partial}{\partial \bar{z}_{l+1}},
$$


its flow is

$$
\varphi_{t}\left(z_{l}, z_{l+1}\right)=\left(e^{t} z_{l}, e^{-t} z_{l+1}\right) .
$$

With respect to $h_{l+1}$, we see

$$
H_{h_{l+1}}=i z_{l} \frac{\partial}{\partial z_{l}}-i \bar{z}_{l} \frac{\partial}{\partial \bar{z}_{l}}+i z_{l+1} \frac{\partial}{\partial z_{l+1}}-i \bar{z}_{l+1} \frac{\partial}{\partial \bar{z}_{l+1}}
$$

Thus, its flow is

$$
\psi_{s}\left(z_{l}, z_{l+1}\right)=\left(e^{i s} z_{l}, e^{i s} z_{l+1}\right)
$$

Now, by considering a new system of E-coordinates, we can refer any focus-focus component for these new E-coordinates, in complex notation, as $\left(z_{l}^{\prime}, z_{l+1}^{\prime}\right)$, and then look at the expressions of the local Hamiltonian vector fields corresponding to $h_{l}^{\prime}$ and $h_{l+1}^{\prime}$. A similar argument referred to the locus of points where such vector fields vanish shows the coincidence up to order of the pair of planes (transverse planes) corresponding to both systems of E-coordinates.

With this argument we have seen that on the level $L_{0}$, and for any two pairs of 'focus-focus' functions corresponding to two systems of E-coordinates around the point $p$, and after reordering it is verified

$$
H_{h_{l}}=a H_{h_{l}^{\prime}}+b H_{h_{l+1}^{\prime}}, \quad H_{h_{l+1}}=c H_{h_{l}^{\prime}}+d H_{h_{l+1}^{\prime}}, \quad l=r+2 k_{h}+1, \ldots
$$

for some constants $a, b, c, d$.

As the vector fields $H_{l+1}^{\prime}$ and $H_{l+1}$ are associated to a Hamiltonian $S^{1}$-action, we have $c=0, d= \pm 1$, we may assume $d=1$.

This means, by using inner contraction with the symplectic two-form, that $\operatorname{Im}\left(d\left(\bar{z}_{l} z_{l+1}\right)\right)=\operatorname{Im}\left(d\left(\bar{z}_{l}^{\prime} z_{l+1}^{\prime}\right)\right)$.

By using once more the inner contraction of $H_{h_{l}}=a H_{h_{l}^{\prime}}+b H_{h_{l+1}^{\prime}}$ with the symplectic two-form we have

$$
\operatorname{Re}\left(d\left(\bar{z}_{l} z_{l+1}\right)\right)=a \cdot \operatorname{Im}\left(d\left(\bar{z}_{l}^{\prime} z_{l+1}^{\prime}\right)\right)+b \cdot \operatorname{Re}\left(d\left(\bar{z}_{l}^{\prime} z_{l+1}^{\prime}\right)\right)
$$

In the plane $z_{l}=0$ we have

$$
\begin{aligned}
& \operatorname{Im}\left(z_{l+1} d \bar{z}_{l}\right)=\operatorname{Im}\left(z_{l+1}^{\prime} d \bar{z}_{l}^{\prime}\right) \\
& \operatorname{Re}\left(z_{l+1} d \bar{z}_{l}\right)=a \cdot \operatorname{Im}\left(z_{l+1}^{\prime} d \bar{z}_{l}^{\prime}\right)+b \cdot \operatorname{Re}\left(z_{l+1}^{\prime} d \bar{z}_{l}^{\prime}\right)
\end{aligned}
$$

In the plane $z_{l+1}=0$ we have

$$
\begin{aligned}
& \operatorname{Im}\left(z_{l} d \bar{z}_{l+1}\right)=\operatorname{Im}\left(z_{l}^{\prime} d \bar{z}_{l+1}^{\prime}\right) \\
& \operatorname{Re}\left(z_{l} d \bar{z}_{l+1}\right)=a \cdot \operatorname{Im}\left(z_{l}^{\prime} d \bar{z}_{l+1}^{\prime}\right)+b \cdot \operatorname{Re}\left(z_{l}^{\prime} d \bar{z}_{l+1}^{\prime}\right)
\end{aligned}
$$

By considering, for instance, the above equations corresponding to the plane $z_{l}=0$, we obtain

$$
\operatorname{Re}\left(z_{l} d \bar{z}_{l+1}\right)=a \cdot \operatorname{Im}\left(z_{l} d \bar{z}_{l+1}\right)+b \cdot \operatorname{Re}\left(z_{l}^{\prime} d \bar{z}_{l+1}^{\prime}\right)=a \cdot \operatorname{Im}\left(z_{l} d \bar{z}_{l+1}\right)+b \cdot \operatorname{Re}\left(z_{l} d \bar{z}_{l+1}\right)
$$

because $\operatorname{Re}\left(z_{l} d \bar{z}_{l+1}\right)=\operatorname{Re}\left(z_{l}^{\prime} d \bar{z}_{l+1}^{\prime}\right)$, thus, we obtain

$$
(1-b) \cdot \operatorname{Re}\left(z_{l} d \bar{z}_{l+1}\right)=a \cdot \operatorname{Im}\left(z_{l} d \bar{z}_{l+1}\right)
$$

so, necessarily $a=0$ and $b=1$. 
We have proved that on the union of planes $\left\{z_{l}=0\right\} \cup\left\{z_{l+1}=0\right\}$ it is verified:

$$
H_{h_{l}}=H_{h_{l}^{\prime}} \quad \text { and } \quad H_{h_{l+1}}=H_{h_{l+1}^{\prime}} .
$$

On $\left\{z_{l}=0=z_{l}^{\prime}\right\}$, this gives

$$
\begin{aligned}
i z_{l+1} \frac{\partial}{\partial z_{l+1}}-i \bar{z}_{l+1} \frac{\partial}{\partial \bar{z}_{l+1}} & =i z_{l+1}^{\prime} \frac{\partial}{\partial z_{l+1}^{\prime}}-i \bar{z}_{l+1}^{\prime} \frac{\partial}{\partial \bar{z}_{l+1}^{\prime}} \\
z_{l+1} \frac{\partial}{\partial z_{l+1}}+\bar{z}_{l+1} \frac{\partial}{\partial \bar{z}_{l+1}} & =z_{l+1}^{\prime} \frac{\partial}{\partial z_{l+1}^{\prime}}+\bar{z}_{l+1}^{\prime} \frac{\partial}{\partial \bar{z}_{l+1}^{\prime}},
\end{aligned}
$$

by taking the inner contraction of the above two terms with the symplectic two form we see

$$
z_{l+1} d \bar{z}_{l}=z_{l+1}^{\prime} d \bar{z}_{l}^{\prime}, \quad \text { on } \quad\left\{z_{l}=0\right\} .
$$

In a similar way, we see that on $\left\{z_{l+1}=z_{l+1}^{\prime}=0\right\}$ we have

$$
i z_{l} \frac{\partial}{\partial z_{l}}-i \bar{z}_{l} \frac{\partial}{\partial \bar{z}_{l}}=i z_{l}^{\prime} \frac{\partial}{\partial z_{l}^{\prime}}-i \bar{z}_{l}^{\prime} \frac{\partial}{\partial \bar{z}_{l}^{\prime}}
$$

which gives

$$
\bar{z}_{l} d z_{l+1}=\bar{z}_{l}^{\prime} d z_{l+1}^{\prime}, \quad \text { on } \quad\left\{z_{l+1}=0\right\} .
$$

By a standard process of integration, one can see that the relations of the above two systems of complex coordinates on $\left\{z_{l}=z_{l}^{\prime}=0\right\} \cup\left\{z_{l+1}=z_{l+1}^{\prime}=0\right\}$ is

$$
\begin{aligned}
& z_{l}=e^{\alpha+i \beta} \cdot z_{l}, \quad \text { for some constants } \alpha \text { and } \beta, \text { on } z_{l+1}=0 \\
& z_{l+1}^{\prime}=e^{\gamma+i \delta} \cdot z_{l+1}^{\prime}, \quad \text { for some constants } \gamma \text { and } \delta, \text { on } z_{l}=0 .
\end{aligned}
$$

Finally, we remark that as the 1 -forms $\bar{z}_{l} d z_{l+1}$ and $\bar{z}_{l}^{\prime} d z_{l+1}^{\prime}$ coincide on $\left\{z_{l+1}=0\right\}$, we have that $d \bar{z}_{l} \wedge d z_{l+1}=d \bar{z}_{l}^{\prime} \wedge d z_{l+1}^{\prime}$ at the point $z_{l}=z_{l+1}=0$, so necessarily $\alpha+\gamma=0$ and $\beta=\delta$.

So, for each focus-focus component, we have seen that the relation, on the level, of two systems of E-coordinates is, up to order, given by

$$
z_{l}^{\prime}=e^{\alpha+i \beta} z_{l}, \quad z_{l+1}^{\prime}=e^{-\alpha+i \beta} z_{l+1} .
$$

So, for any two systems of E-coordinates, we know the coincidence of the pairs of axes corresponding to the hyperbolic components, and the coincidence of the pairs of planes corresponding to the focus-focus components; we are going to see that the regular parts also coincide. To prove it we have to recall that on the level $L_{0}$, the relations between the corresponding hyperbolic and focus-focus components are given, as we have just seen, by

$$
x_{i}^{\prime}=b_{i} x_{i}, \quad y_{i}^{\prime}=\frac{1}{b_{i}} y_{i}, \quad b_{i} \quad \text { constant }, \quad i=r+1, \ldots, r+k_{h}
$$


for each hyperbolic component, and

$$
z_{l}^{\prime}=e^{\alpha_{l}+i \beta_{l}} z_{l}, \quad z_{l+1}^{\prime}=e^{-\alpha_{l}+i \beta_{l}} z_{l+1}, \quad \alpha_{l} \quad \text { and } \quad \beta_{l} \quad \text { constants, } \quad l=r+2 k_{h}+1, \ldots
$$

for each focus-focus component.

As a direct consequence of this fact, we have that the regular parts of the level, for both systems of E-coordinates are just the same. Of course, we can use the same functions $y_{1}, \ldots, y_{r}$ for both systems of E-coordinates.

As the singular composition of each point is, in fact, a product of regular, hyperbolic and focus-focus components, and we know the uniqueness up to order of this decomposition, we have to show now how this identification is done in hyperbolic and focus-focus cases. In the regular case the identification used will be trivial. To explain it in a detailed form, we start by considering two simple type of singularities, the hyperbolic and focus-focus cases, and finally we consider the general case.

3.3.1. Hyperbolic identification. Let $\left(p,\left[V_{1}\right]\right),\left(p,\left[V_{2}\right]\right) \in \hat{L}_{0}$, where $p \in L_{0}$ is a purely hyperbolic point (degree of hyperbolicity equal to one). Then in Eliasson coordinates $(x, y)$ around the point $p$ in $M$, we may assume $\left[V_{1}\right]=\left[\left(\frac{\partial}{\partial x}\right)\right]$ and $\left[V_{2}\right]=\left[\left\langle\frac{\partial}{\partial y}\right\rangle\right]$. Recalling Claim 2.1, we shall use $x$ and $y$ as a system of coordinates in the chosen neighborhoods of $\left(p,\left[V_{1}\right]\right)$ and $\left(p,\left[V_{2}\right]\right)$ in $\hat{L}_{0}$.

We take the corresponding canonical coordinates in $T^{*} \hat{L}_{0}$ in two neighborhoods $U_{1}$ of $\left(\left(p,\left[V_{1}\right]\right), 0\right), U_{2}$ of $\left(\left(p,\left[V_{2}\right]\right), 0\right)$, and we denote them by $\left(x, x^{*}\right)$ and $\left(y, y^{*}\right)$, respectively. Looking at the local expressions, that we have found previously for each function $g_{i}$, the basic functions will depend on $x x^{*}$ and $y y^{*}$, respectively, and the canonical symplectic two forms are $d x \wedge d x^{*}$ and $d y \wedge d y^{*}$, respectively.

So, the symplectomorphism from $U_{1}$ onto $U_{2}$, we are searching for, is expressed in these coordinates by

$$
\left\{\begin{array}{l}
y=-x^{*} \\
y^{*}=x
\end{array} .\right.
$$

Note that this identification is determined by the fact that $\omega_{0}\left(\frac{\partial}{\partial x}, \frac{\partial}{\partial y}\right)>0$.

We have to check that this identification does not depend on the system of Eliasson coordinates that we have chosen. At this point, we observe that two systems of Ecoordinates $(x, y)$ and $\left(x^{\prime}, y^{\prime}\right)$ around $p$, and restricted to the axes $\{x=0\} \cup\{y=0\}$ are related unless a rotation of angle $n \frac{\pi}{2}$, by a change of the form

$$
\left\{\begin{array}{l}
x^{\prime}=b \cdot x \\
y^{\prime}=\frac{1}{b} \cdot y
\end{array}\right.
$$

and one can check easily that the above gluing is well defined, i.e. it is independent on the E-coordinates that we have chosen.

3.3.2. Focus-focus identification. We can proceed in a similar way for two points $\left(p,\left[V_{1}\right]\right),\left(p,\left[V_{2}\right]\right)$, in the preimage of a focus-focus point $p \in L_{0}$. Regarding to the proof 
of Claim 2.1 one can consider

$$
\left[V_{1}\right]=\left[\left\langle\frac{\partial}{\partial x_{1}}, \frac{\partial}{\partial x_{2}}\right\rangle\right], \quad\left[V_{2}\right]=\left[\left\langle\frac{\partial}{\partial y_{1}}, \frac{\partial}{\partial y_{2}}\right\rangle\right]
$$

We take canonical coordinates in $T^{*} \hat{L}_{0}$ in two neighborhoods $U_{1}$ of $\left(p,\left[V_{1}\right]\right)$ and $U_{2}$ of $\left(p,\left[V_{2}\right]\right)$, and we denote them by $\left(x_{1}, x_{2}, x_{1}^{*}, x_{2}^{*}\right)$ and $\left(y_{1}, y_{2}, y_{1}^{*}, y_{2}^{*}\right)$, respectively. As in the hyperbolic case, and attending to the local expressions of the functions $g_{i}$, the basic functions depend on $x_{1} x_{1}^{*}+x_{2} x_{2}^{*},-x_{1}^{*} x_{2}+x_{1} x_{2}^{*}$ and $y_{1} y_{1}^{*}+y_{2} y_{2}^{*},-y_{1}^{*} y_{2}+y_{1} y_{2}^{*}$, respectively. So, the symplectomorphism from $U_{1}$ to $U_{2}$ we need is given by

$$
\left\{\begin{array}{l}
y_{1}=-x_{1}^{*} \\
y_{2}=-x_{2}^{*} \\
y_{1}^{*}=x_{1} \\
y_{2}^{*}=x_{2}
\end{array} .\right.
$$

By using complex notation, as we made in the above considerations, we can take $z_{1}=x_{1}+i x_{2}, z_{2}=y_{1}+i y_{2}$. We know that

$$
\begin{aligned}
h_{1}+i h_{2} & =\bar{z}_{1} \cdot z_{2} \\
\omega_{0} & =\operatorname{Re} \quad d \bar{z}_{1} \wedge d z_{2}
\end{aligned}
$$

The Lagrangian foliation is given by the level surfaces of

$$
\bar{z}_{1} \cdot z_{2} \text {. }
$$

The level through the point $(0,0)$ is the union of the planes $\left\{z_{1}=0\right\}$ and $\left\{z_{2}=0\right\}$. The neighborhoods $U_{1}$ and $U_{2}$ are neighborhoods of the origin at the planes $\left\{z_{2}=0\right\}$ and $\left\{z_{1}=0\right\}$, respectively. The identification map we consider is given by

$$
\left\{\begin{array}{l}
z_{2}=-z_{1}^{*} \\
z_{2}^{*}=z_{1}
\end{array}\right.
$$

Note that, as in the hyperbolic case, this identification is determined by the fact that $\omega_{0}\left(\frac{\partial}{\partial \bar{z}_{1}}, \frac{\partial}{\partial z_{2}}\right)>0$.

As in the hyperbolic case, we have to check that this identification does not depend on the chosen system of E-coordinates.

We recall that the relations of the E-coordinates on the level in this case is given by

$$
z_{1}^{\prime}=e^{\alpha+i \beta} z_{l}, \quad z_{2}^{\prime}=e^{-\alpha+i \beta} z_{2}, \quad \alpha \quad \text { and } \beta \text { constants, }
$$

and one can check easily that the above gluing is well defined, i.e. it is independent on the E-coordinates that we have chosen.

3.3.3. General identification. Let $p$ be a point of $L_{0}$ of Williamson type $\left(k_{h}, k_{f}\right)$. Any system of E-coordinates around this point consists of $r$ regular terms; following the notation introduced in Section 2.1, there are $r$ functions $h_{i}=y_{i}$, defined in a regular block described by the coordinates $\left(x_{1}, y_{1}, \ldots, x_{r}, y_{r}\right)$. There are $k_{h}$ hyperbolic function $h_{i}=x_{i} \cdot y_{i}$, this gives $k_{h}$ hyperbolic blocks, described by the coordinates $\left(x_{i}, y_{i}\right)$. Finally, 
there are $2 k_{f}$ functions of the form $h_{i}=x_{i} y_{i}+x_{i+1} y_{i+1}, h_{i+1}=x_{i} y_{i+1}-y_{i} x_{i+1}$, this gives $k_{f}$ focus-focus blocks described by the coordinates $\left(x_{i}, x_{i+1}, y_{i}, y_{i+1}\right)$.

According to this, we have a local description (in a neighborhood of $p$ ) of the affine structure on a neighborhood of $p$ in $L_{0}$, by considering the restriction to $L_{0}$ of the local Hamiltonian vector fields corresponding to the functions $h_{i}$. These Hamiltonian vector fields are

$\frac{\partial}{\partial x_{i}} \quad$ in the regular case,

$x_{i} \frac{\partial}{\partial x_{i}}-y_{i} \frac{\partial}{\partial y_{i}}$ in the hyperbolic case,

and the pair of vector fields $x_{i} \frac{\partial}{\partial x_{i}}+x_{i+1} \frac{\partial}{\partial x_{i+1}}-y_{i} \frac{\partial}{\partial y_{i}}-y_{i+1} \frac{\partial}{\partial y_{i+1}}$

$-x_{i+1} \frac{\partial}{\partial x_{i}}+x_{i} \frac{\partial}{\partial x_{i+1}}-y_{i+1} \frac{\partial}{\partial y_{i}}+y_{i} \frac{\partial}{\partial y_{i+1}}$ in the focus-focus case.

Now let $\hat{p}$ be a point of $j^{-1}(p)$. Recall that $j^{-1}(p)$ contains $2^{k_{h}+k_{f}}$ points, and we have a branch of $\hat{L}_{0}$ through any one of these points. Following Section 2.2 we can express any point $\hat{p} \in j^{-1}(p)$ as $\hat{p}=(p,[V])$,

$$
V=\bigoplus_{i=1}^{r}\left\langle\frac{\partial}{\partial x_{i}}\right\rangle \bigoplus_{i=r+1}^{r+k_{h}}\left\langle\frac{\partial}{\partial \alpha_{i}}\right\rangle \bigoplus_{i=r+k_{h}+1}^{r+k_{h}+2 k_{f}-1}\left\langle\frac{\partial}{\partial \beta_{i}}, \frac{\partial}{\partial \beta_{i+1}}\right\rangle
$$

Any term $\frac{\partial}{\partial \alpha_{i}}$ in a hyperbolic element means either $\frac{\partial}{\partial x_{i}}$ or $\frac{\partial}{\partial y_{i}}$. By a similar convention, any pair $\frac{\partial}{\partial \beta_{i}}, \frac{\partial}{\partial \beta_{i+1}}$ is either $\frac{\partial}{\partial x_{i}}, \frac{\partial}{\partial x_{i+1}}$ or $\frac{\partial}{\partial y_{i}}, \frac{\partial}{\partial y_{i+1}}$.

The system of coordinates to take in the branch of $\hat{L}_{0}$ through $\hat{p}$ is

$$
\left(x_{1}, \ldots, x_{r} ; \alpha_{r+1}, \ldots, \alpha_{r+k_{h}} ; \beta_{r+k_{h}+1}, \ldots, \beta_{r+k_{h}+2 k_{k}}\right) \text {. }
$$

REMARK 3.1. The branch through $\hat{p}$ is the product of an open neighborhood of 0 in $\mathbb{R}^{r}$ (the regular part of the branch) with $k_{h}$ copies of an open interval of $\mathbb{R}$, which are the hyperbolic elements, and finally $k_{f}$ copies of an open neighborhood of 0 in $\mathbb{R}^{2}$, which are the focus-focus elements.

Note that the affine structure of the branch is described by the vector fields

$$
\begin{aligned}
& \frac{\partial}{\partial x_{i}}, \quad i=1, \ldots, r \quad \text { the regular part } \\
& x_{i} \frac{\partial}{\partial x_{i}} \quad \text { or } \quad-y_{i} \frac{\partial}{\partial y_{i}}, \quad i=r+1, \ldots, r+k_{h} \quad \text { the hyperbolic part. }
\end{aligned}
$$

And corresponding to the focus-focus part, we have one of the two pairs of vector fields

$$
\begin{aligned}
x_{i} & \frac{\partial}{\partial x_{i}}+x_{i+1} \frac{\partial}{\partial x_{i+1}}, \quad-x_{i+1} \frac{\partial}{\partial x_{i}}+x_{i} \frac{\partial}{\partial x_{i+1}} \text { or } \\
& -y_{i} \frac{\partial}{\partial y_{i}}-y_{i+1} \frac{\partial}{\partial y_{i+1}}, \quad-y_{i+1} \frac{\partial}{\partial y_{i}}+y_{i} \frac{\partial}{\partial y_{i+1}}, \quad i=r+k_{h}+1, \ldots, r+k_{h}+2 k_{f}-1 .
\end{aligned}
$$


Let us define now the local identification between two branches of $\hat{L}_{0}$ corresponding to two points $\hat{p}_{1}, \hat{p}_{2} \in j^{-1}(p)$. Each one of these branches is a product of regular, hyperbolic and focus-focus components. The identification that we are searching for will act trivially on the regular component. For the common hyperbolic elements, the mapping to consider is obviously trivial, and if not the map is given by $y_{i}=-x_{i}^{*}, y_{i}^{*}=$ $x_{i}$. For the focus-focus elements we proceed in a similar form: we take the identity if they coincide and if not we take the map given by

$$
y_{i}=-x_{i}^{*}, \quad y_{i+1}=-x_{i+1}^{*}, \quad y_{i}^{*}=x_{i}, \quad y_{i+1}^{*}=x_{i+1} .
$$

REMARK 3.2. Note that the regular part and each one of the hyperbolic and focus-focus elements are completely determined by the affine structure on $L_{0}$.

\section{Note that this last remark ensures the compatibility of the local identifications defined.}

At this point, we remark that the local expressions of the functions $g_{i}$ (look at (1) in Section 3.2), are preserved by these gluings.

Summarizing, the gluing is well defined, and we remark that the local expressions of the functions $g_{i}$ (look at (1) in Section 3.2), are preserved by these gluings.

Once we have shown how to identify the neighborhoods of the points in the same preimage we get a germ of $2 n$-dimensional symplectic manifold $\left(N, \omega_{0}\right)$ containing $L_{0}$ as a singular Lagrangian submanifold. We have just seen that that the functions $g_{i}$, $i=1, \ldots, n$, can be projected to $N$. Let $G_{1}, \ldots, G_{n}$ be the projected functions.

So, we have proved

THEOREM 3.1. $\left(N, \omega_{0},\left(G_{1}, \ldots, G_{n}\right)\right)$ is a completely integrable system, having $L_{0}$ as a singular level, and such that the singular affine structure on $L_{0}$ is the initial one. This is the c.i.s. that we say the linearized c.i.s. of the given $\left(M^{2 n}, \omega,\left(f_{1}, \ldots f_{n}\right)\right)$.

3.4. Construction of c.i.s. with prescribed singularities. As a sort of application of the above considerations, let us point out how to give some c.i.s. with prescribed singularities, around a singular level.

The intrinsic geometry of the level, i.e. the number and kind of singular points, is obviously related with the kind of singularities along its singular points.

We show how to obtain a c.i.s. around a 2-dimensional singular level with a focusfocus point and one circle of hyperbolic points:

Let us consider the 2-sphere $S^{2}$. This manifold $S^{2}$ will play the role of the desingularized level, $\hat{L}_{0}$, of the above sections. We shall consider $T^{*} S^{2}$ and two vector fields, with singularities, which will be used to define two functions on $T^{*} S^{2}$. These functions have singularities at several points, and by furnishing the local identifications at the corresponding singular points, we will get the c.i.s. around the singular level.

Let $\theta$ (longitude) and $\varphi$ (latitude) be polar coordinates on $S^{2}$.The vector fields to consider are: $X=\frac{\partial}{\partial \theta}$ and $Y=h(\varphi) \frac{\partial}{\partial \varphi}$. We have to give a "good"expression for $h(\varphi)$, obviously we take $h\left(-\frac{\pi}{2}\right)=0, h\left(\frac{\pi}{2}\right)=0$, and $h\left(-\frac{\pi}{4}\right)=h\left(\frac{\pi}{4}\right)=0$. We consider four open 
subsets of $S^{2}$

$$
\begin{aligned}
& U_{1}=\left\{(\theta, \varphi) \quad \mid \quad-\frac{\pi}{2} \leq \varphi<-\frac{\pi}{2}+\varepsilon\right\}, \\
& V_{1}=\left\{(\theta, \varphi) \quad \mid \quad-\frac{\pi}{4}-\varepsilon<\varphi<\frac{\pi}{4}+\varepsilon\right\}, \\
& V_{2}=\left\{(\theta, \varphi) \quad \mid \quad \frac{\pi}{4}-\varepsilon<\varphi<\frac{\pi}{4}+\varepsilon\right\}, \\
& U_{2}=\left\{(\theta, \varphi) \quad \mid \quad \frac{\pi}{2}-\varepsilon<\varphi \leq \frac{\pi}{2}\right\} \text {, }
\end{aligned}
$$

where $\varepsilon<\frac{\pi}{8}$.

On $U_{1}$ and $U_{2}$ we can take as coordinates the first two cartesian coordinates $\left(x_{1}, x_{2}\right)$, and consider the vector fields:

on $U_{1}, x_{1} \frac{\partial}{\partial x_{1}}+x_{2} \frac{\partial}{\partial x_{2}}=-\frac{\cos \varphi}{\sin \varphi} \cdot \frac{\partial}{\partial \varphi}$ and $-x_{2} \frac{\partial}{\partial x_{1}}+x_{1} \frac{\partial}{\partial x_{2}}=\frac{\partial}{\partial \theta}$,

on $U_{2},-x_{1} \frac{\partial}{\partial x_{1}}-x_{2} \frac{\partial}{\partial x_{2}}=\frac{\cos \varphi}{\sin \varphi} \cdot \frac{\partial}{\partial \varphi}$ and $-x_{2} \frac{\partial}{\partial x_{1}}+x_{1} \frac{\partial}{\partial x_{2}}=\frac{\partial}{\partial \theta}$.

On $V_{1}$ and $V_{2}$ we can take $(\theta, \varphi)$ as coordinates and the following vector fields:

on $V_{1},-\left(\varphi+\frac{\pi}{4}\right) \frac{\partial}{\partial \varphi}$ and $\frac{\partial}{\partial \theta}$,

on $V_{2},\left(\varphi-\frac{\pi}{4}\right) \frac{\partial}{\partial \varphi}$ and $\frac{\partial}{\partial \theta}$.

Now we see that the function $h(\varphi)$ we are searching for can be a differentiable function of $\varphi$, such that its values in $U_{1}, V_{1}, U_{2}, V_{2}$ are the above prescribed and not vanishing on $S^{2} \backslash\left(U_{1} \cup V_{1} \cup U_{2} \cup V_{2}\right)$.

Finally, we consider on $T^{*} S^{2}$ the pair of functions $f, g$ associated to the vector fields $X, Y$, defined as follows: for any point $(z, w) \in T^{*} S^{2}, f(z, w):=$ $\langle X(z), w\rangle, g(z, w):=\langle Y(z), w\rangle$. The c.i.s. we are searching for is obtained by a process of gluing from $\left(T^{*} S^{2}, \omega_{0},(f, g)\right)$. This gluing can be easily established by defining two symplectomorphisms: one of them is a local symplectomorphism between $\left(T^{*} U_{1},\left(x_{1}=0, x_{2}=0,0,0\right)\right)$ and $\left(T^{*} U_{2},\left(X_{1}=0, X_{2}=0,0,0\right)\right)$. We recall (see hyperbolic gluing in Section 3.2) that the mapping is given by

$$
\left(x_{1}, x_{2}, y_{1}, y_{2}\right) \longmapsto\left(X_{1}=-y_{1}, X_{2}=-y_{2}, Y_{1}=x_{1}, Y_{2}=x_{2}\right)
$$

The other symplectomorphism we need to realize the gluing is a semilocal symplectomorphism from $\left(T^{*} V_{1},\left(\theta, \varphi=-\frac{\pi}{4}, 0,0\right)\right)$ in $\left.T^{*} V_{2},\left(\theta, \varphi=\frac{\pi}{4}, 0,0\right)\right)$, described as follows: on $T^{*} V_{1}$ we take $\left(\theta, \varphi+\frac{\pi}{4}, \Theta, \Phi\right)$ as canonical coordinates. In the same form, we take $\left(\bar{\theta}=\theta, \bar{\varphi}-\frac{\pi}{4}=\varphi-\frac{\pi}{4}, \bar{\Theta}=\Theta, \bar{\Phi}\right)$ as canonical coordinates in $T^{*} V_{2}$. The symplectomorphism we need to define the gluing is

$$
\left(\theta, \varphi+\frac{\pi}{4}, \Theta, \Phi\right) \longmapsto\left(\bar{\theta}=\theta, \bar{\varphi}-\frac{\pi}{4}=\Phi, \bar{\Theta}=\Theta, \bar{\Phi}=-\left(\varphi+\frac{\pi}{4}\right)\right)
$$

Thus, the quotient of a germ of neighborhood of $S^{2}$ in $T^{*} S^{2}$, by using these identifications provides us a germ of completely integrable system around a level having one singular point of focus-focus type and one circle of hyperbolic points.

One sees from this construction that the same arguments can serve to give c.i.s. with several points of focus-focus type; it should be necessary to use different copies of $S^{2}$ and define a gluing by using the poles alternatively. The above construction suggests different ways of having circles of hyperbolic points in the level. 
ACKNOWLEDGEMENTS. Partially supported by the DGICYT/FEDER project MTM2009-07594: Estructuras Geometricas: Deformaciones, Singularidades y Geometria Integral, and by the PIGC-AGAU, 2009SGR119: Grup de Recerca en Geometria i Topologia Algebraica.

\section{REFERENCES}

1. V. I. Arnold, Mathematical methods of classical mechanics, 2nd ed., Graduate Texts in Mathematics, vol. 60 (Springer-Verlag, New York, 1989).

2. M. Audin, A. Cannas da Siva and E. Lerman,Symplectic geometry of integrable systems, Advanced Courses in Mathematics (CRM Barcelona, Birkhäuser, 2003).

3. A. V. Bolsinov and A. T. Fomenko, Integrable Hamiltonian systems, geometry, topological classification (Chapman \& Hall/CRC, 2004).

4. M. Boucetta and P. Molino, Géométrie globale des systèmes Hamiltoniennes complétement intégrables, C. R. Acad. Sci. Paris, I 308(13) (1989), 421-424.

5. C. Currás-Bosch, Decomposition of integrable and non-degenerate Hamiltonian systems with non-zero ellipticity degree, preprint.

6. C. Currás-Bosch and P. Molino, Holonomie, suspensions et classifications pour les feuilletages Lagrangiens, C. R. Acad. Sci. Paris, I 32611 (1989), 1317-1320.

7. T. Delzant, Hamiltoniens periodiques et images convexes de l'application moment, Bull. Soc. Math. France 116(3) (1988), 315-339.

8. J. J. Duistermaat, On global action-angle coordinates, Commun. Pure Appl. Math. 33(6) (1980), 687-706.

9. L. H. Eliasson, Normal forms for Hamiltonian systems with Poisson commuting integrals, PhD Thesis (University of Stockholm, 1984).

10. A. T. Fomenko, Topological classification of integrable systems, in Advances in Soviet Mathematics, vol. 6 (AMS, Providence R. I., 1991).

11. E. Lerman and Y. Umanskii, Classification of 4-dimensional integrable Hamiltonian systems, in Methods of qualitative theory of bifurcations (Iz.Garkov University, Gorki, 1988), $67-76$.

12. H. Mineur, Réduction des systèmes mécaniques à $\mathrm{n}$ degrès de liberté, J. Math. Pure Appl. IX, 15 (1936), 385-389.

13. E. Miranda, On symplectic linearization of singular Lagrangian foliations (Tesi University de Barcelona, 2003). $27-45$.

14. E. Miranda and V. N. San, A singular Poincaré Lemma, Int. Math. Res. Notices (2005),

15. E. Miranda and N. T. Zung, Equivariant normal forms for non-degenerate singular orbits of integrable Hamiltonian systems, Ann. Sci. Ec. Norm. Sup., 37 (2004), 819-839.

16. $\mathrm{P}$. Molino, Action-angle with singularities and non-degenerate integrable systems, (preprint).

17. A. Toulet, Classification des systèmes intégrables en dimension 2 (Thèse University Montpellier II, 1996).

18. V. N. San, On semi-global invariants for focus-focus singularities, Topology 42 (2003), $365-380$

19. N. T. Zung, Symplectic topology of integrable Hamiltonian systems $\mathrm{PhD}$ Thesis (University of Strasbourg, 1994).

20. N. T. Zung, Symplectic topology of integrable Hamiltonian systems I, Compos. Math. 101 (1996), 179-219.

21. N. T. Zung, Symplectic topology of integrable Hamiltonian systems II, Compos. Math. 138 (2003), 125-156.

22. A. Weinstein, Lectures on symplectic manifolds, Regional Conference Series in Mathematics (American Mathematical Society, Providence, RI, 1976).

23. J. Williamson, On the algebraic problem concerning the normal form of linear dynamical systems, Am. J. Math. 58(1) (1936), 141-163. 